OUTP-07-14-P

LAPTH-1219/2007

\title{
Recent results on unintegrated parton distributions
}

\author{
F. Hautmann ${ }^{1,2}$ and H. Jung ${ }^{3}$ \\ ${ }^{1}$ Oxford University, Department of Theoretical Physics, Oxford OX1 3NP \\ ${ }^{2}$ LAPTH, F-74941 Annecy-le-Vieux \\ ${ }^{3}$ Deutsches Elektronen Synchrotron, D-22603 Hamburg
}

\begin{abstract}
We summarize recent results on i) the use of u-pdf's in the Monte-Carlo simulation of hadronic final states at high-energy colliders, and ii) attempts to characterize u-pdf's with precision in terms of nonlocal operator matrix elements.
\end{abstract}

Presented at the Photon2007 International Conference

Paris 9-13 July 2007, La Sorbonne 


\title{
Recent results on unintegrated parton distributions
}

\author{
F. Hautmann ${ }^{1,2}$ and H. Jung ${ }^{3}$ \\ 1- Oxford University, Department of Theoretical Physics, Oxford OX1 3NP \\ 2- LAPTH, F-74941 Annecy-le-Vieux \\ 3- Deutsches Elektronen Synchrotron, D-22603 Hamburg
}

\begin{abstract}
We summarize recent results on i) the use of u-pdf's in the Monte-Carlo simulation of hadronic final states at high-energy colliders, and ii) attempts to characterize u-pdf's with precision in terms of nonlocal operator matrix elements.
\end{abstract}

\section{Introduction}

At forthcoming high-energy colliders a large amount of events are characterized by complex final states with multiple hard scales, possibly far apart from each other. QCD methods to address multi-scale hadronic processes involve the use of parton distributions unintegrated in both longitudinal and transverse components of parton's momentum (updf's). Classic examples are provided by $\mathrm{Su}-$ dakov physics [1, 2] and small-x physics [3, 4. U-pdf's also govern exclusive processes [5] and spin 6. They are required, in general, to explore detailed features of hadronic final states 7]. For these reasons, theoretical and phenomenological studies of u-pdf's are being actively pursued.

In the case of small x, u-pdf's can be introduced in a gauge-invariant manner using high-energy factorization 8. This result was used early on both for Monte-Carlo simulations 9] of $\mathrm{x} \rightarrow 0$ parton showers and for numerical resummation programs [10] for ln $\mathrm{x}$ corrections to QCD evolution equations. For structure function's evolution, methods are being developed ([11-15] and references therein) to match the $\mathrm{k}_{\perp}$-dependent, small$\mathrm{x}$ dynamics with perturbative collinear dynamics. For the full simulation of exclu- sive components of hadronic final states, on the other hand, such matching is more complex (see discussions in 7 and [16) and is not yet available. This will be critical for turning present event generators based on $\mathrm{u}-$ pdf's [17-23] into general-purpose MonteCarlo tools. Importantly, for $\mathrm{x} \rightarrow 0 \mathrm{u}-$ pdf's may provide a useful framework also for discussing the approach to the saturation regime: see e.g. [24, 25] for recent studies.

In the general case, to characterize u-pdf's gauge-invariantly over the whole phase space is more difficult. Open questions on this issue are the subject of much current activity, see e.g. 26]-40, with wide-ranging applications from semi-inclusive processes to partonshower algorithms to spin physics.

In this report we concentrate on two topics. In Sec. 2 we consider $\mathrm{x} \ll 1$ and discuss recent results from $\mathrm{k}_{\perp}$ Monte-Carlos on the jet structure of small-x final states. In Sec. 3 we describe ongoing progress towards general operator definitions for u-pdf's, focusing on results for the treatment of $\mathrm{x} \rightarrow 1$ endpoint divergences.

\section{Jet final states from uninte- grated parton distributions}

There have recently been new calculations of hadronic jet final states from Monte-Carlo generators based on unintegrated parton distributions.

\subsection{U-pdf's and shower Monte-Carlo generators}

The idea common to the Monte-Carlo event generators based on u-pdf's is to use factor- 
ization at fixed $k_{\perp}[8]$ in order to a) generate the hard scattering event, including dependence on the initial transverse momentum, and b) couple this to the evolution of the initial state to simulate the gluon cascade. Event generators of this kind include SMALLX 9, CASCADE 20, LDCMC 22, and the newer tools 17 and 18. Different generators employ different models for the evolution of the initial state, such as BFKL 41, 42, CCFM 43, 44, LDC 45 evolution. With suitable constraints on the angular ordering of gluon emissions, these can all be set up to give the correct leading $\ln x$ behavior. A number of subleading contributions is however also of importance for realistic simulations of final states. The general approach of these Monte-Carlos and aspects of the differences among them are reviewed in [16. See also 7] for further references.

Although none of the above generators are nearly as developed as standard MonteCarlos like PYTHIA 46 or HERWIG 47, they have the potential advantage of a better treatment of high-energy logarithmic effects. Implementing these effects in the shower can be relevant for the simulation of complex final states at LHC energies, see e.g. multijet studies in 48, and additional references in [7. A further potential advantage is that Monte-Carlos with u-pdf's likely provide a more natural framework 49 to simulate the $\mathrm{k}_{\perp}$ distribution of the soft underlying event [50] (minijets, soft hadrons) and, possibly, multiple interactions at very high energies.

On the other hand, present Monte-Carlos of this kind do not automatically include contributions from collinear radiation associated to $\mathrm{x} \sim 1$. They need to be corrected for this. This is done partially in present implementations, but not yet in a systematic fashion. A recent example of a study in this direction is in [17, based on the procedure of [51, 52.

A related issue concerns the inclusion of quark contributions in the initial state. In present implementations these are either ne- glected or approximated by lowest-order perturbative evolution [17, [18, 22]. The $\mathrm{k}_{\perp-}$ dependent kernel that governs small-x seaquark evolution to all orders is given in [53]. Universality properties of this kernel are emphasized in [12, 54]. The quark kernel is not yet implemented in current Monte-Carlos.

Keeping in mind these limitations, MonteCarlo event generators with u-pdf's can be used at present within the low-x domain to probe the physical picture of space-like $\mathrm{x} \rightarrow 0$ parton showers. A selection of recent results from these event generators is given in the next subsections, going from more inclusive to less inclusive measurements.

\subsection{Inclusive cross sections}

Available $\mathrm{k}_{\perp}$ Monte-Carlos have been used to analyze data for DIS structure functions and for inclusive lepto- and hadro-production of jets, heavy flavors and prompt photons, see [7, 16. These inclusive analyses serve to test the overall consistency of the physical picture and to perform first determinations of the unintegrated gluon distribution.

A consistent semi-quantitative picture is achieved with sensible results for the evolved unintegrated gluon distribution 16, 19, 51, 55, 56, 57, 58. However, the gluon at low scales and low $\mathrm{x}$ is only poorly constrained by this approach [58, 59. This is not unexpected, as the summation of higher orders implied by the Monte-Carlo should reduce the sensitivity of predictions at high scales to the form of the input 60].

Examples of results for jet production in $e p$ and $p \bar{p}$ collisions are reported in Figs. 1 and 2 Fig. 1 shows the description of the jet $E_{T}$ distribution data from $\mathrm{H} 1$ 61 obtained with CASCADE using the recent u-pdf fits 62. Fig. 2] shows the comparison of CDF jet data [63] with the Monte-Carlo [17.

Current uncertainties on the unintegrated gluon are large especially in the lowest $\mathrm{x}$ region 16. Implications of this are studied for heavy-quark structure functions at 


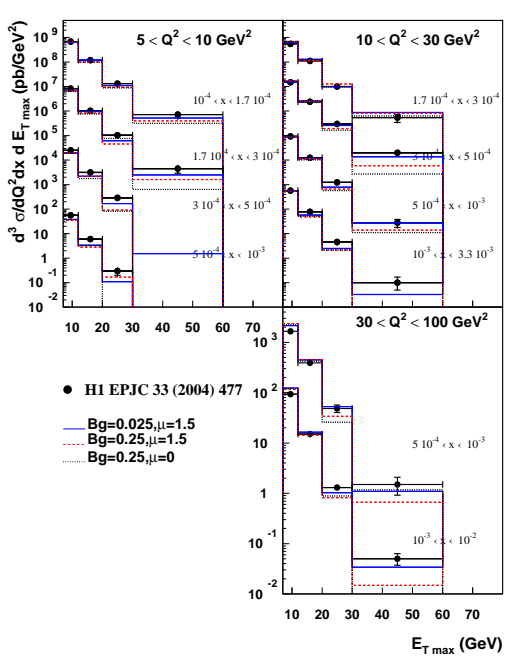

Figure 1: Jet $E_{T}$ distribution at HERA from the $\mathrm{k}_{\perp}$ Monte-Carlo CASCADE and u-pdf fits 62, compared with H1 data 61].

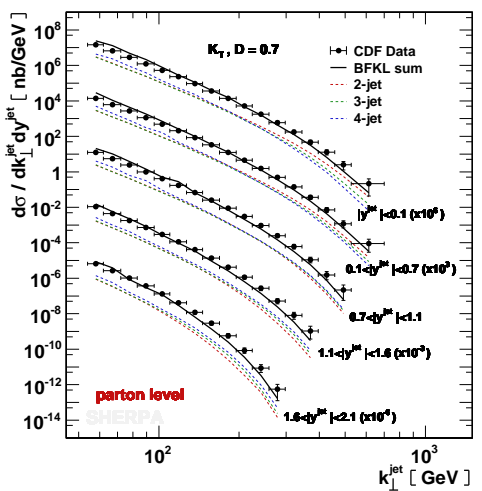

Figure 2: Tevatron jet spectra from [17] compared with $\mathrm{CDF}$ data 63].

HERA in 58 and for jet production at RHIC in 57. Note that first fits of both the $x$ - and $\mathrm{k}_{\perp}$-dependence of the unintegrated gluon are performed in 62.

A potentially important application of these results is to final states containing Higgs bosons at the LHC. The Higgs matrix element coupled to the unintegrated gluon distribution is computed in 64]. First results from Monte-Carlo implementation are given in 19. Studies of the Higgs transversemomentum spectrum from NLO + soft-gluon resummation 65, 66 indicate that small-x terms, although highly subleading from the viewpoint of the soft hierarchy, appear to affect the spectrum at a level comparable to the current theoretical uncertainties estimated as in 67. Further investigations of these effects 22, 68, 69, 70, 71 are warranted. An additional, special application is to central exclusive production, recently considered for Higgs [72, 73] and scalar $\chi$ mesons [74] in the context of u-pdf's.
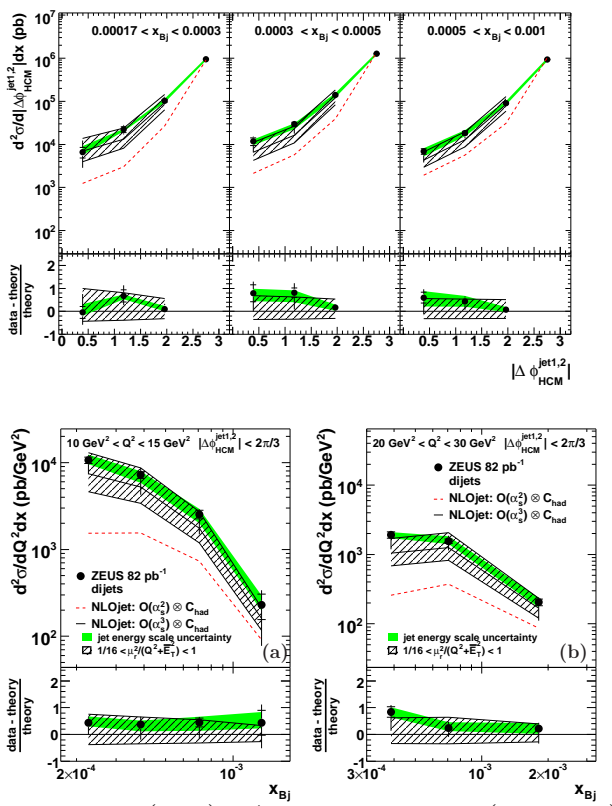

Figure 3: (top) Azimuth and (bottom) Bjorken-x dependences of di-jet distributions measured by Zeus 78 .

We conclude this subsection by observing that recently the small-x definition [8] of $\mathrm{u}-$ pdf's has also been used [75, 76] to treat nonleading corrections to jet production at large rapidity separations in hadron-hadron collisions [77, and Monte-Carlo event generators based on the evolution 41,42 are also becoming available for these processes.

\section{$2.3 \quad$ Multi-jet correlations}

More details of the parton $\mathrm{k}_{\perp}$ dynamics can be probed by examining jet correlations in final states containing multiple jets. 
Recently the Zeus collaboration has presented measurements of two-jet and three-jet distributions in DIS associated with low $\mathrm{x}$, $10^{-4}<x<10^{-2}[78$. Zeus' jet definition is such that nonglobal logarithms from jet clustering are avoided [79, as are double logarithms from symmetric cuts 80 . Fig. 3 [78 shows two-jet data versus the azimuthal separation $\Delta \phi$ and versus Bjorken $\mathrm{x}$, compared with next-to-leading-order perturbative results 81. Notice the large variation from the order- $\alpha_{s}^{2}$ to the order- $\alpha_{s}^{3}$ result with decreasing $x$ and decreasing $\Delta \phi$.
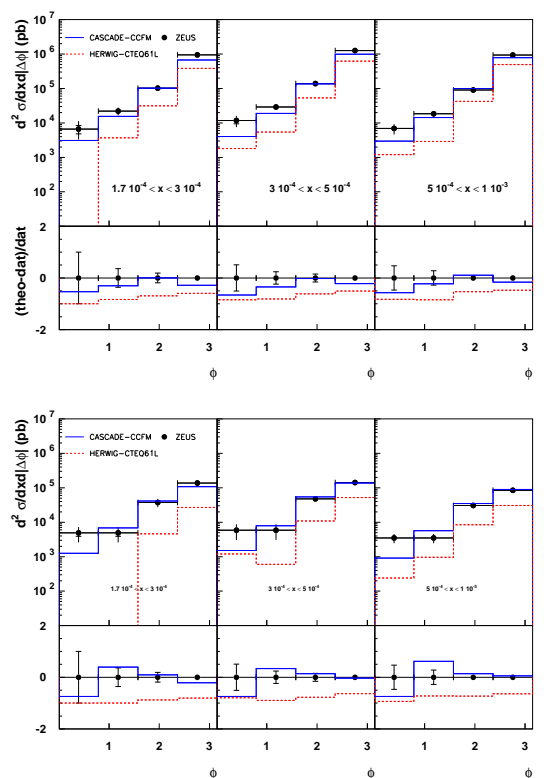

Figure 4: Angular jet correlations 60 from CASCADE and HERWIG compared with Zeus data [78: (top) di-jet cross section; (bottom) three-jet cross section.

Ref. 60 analyzes the multi-jet final states in the framework of u-pdf's. In particular, it studies the contribution to potentially large higher-order corrections arising from a sizeable $\mathrm{k}_{\perp}$ in the initial state when several wellseparated hard jets are produced. In this kinematics, effects from the $\mathrm{x} \ll 1$ parton shower may be enhanced. These contributions are important at small $\Delta \phi$, when the jets are not close to back-to-back configura- tions 82 . Fig. 4 4 shows results for the distribution in the azimuthal separation between the leading jets 60, compared with the measurement [78. Fig. [5 60] shows the angular distribution for the third jet, in the cases of small separations and large separations between the leading jets. The $\mathrm{k}_{\perp}$ Monte-Carlo
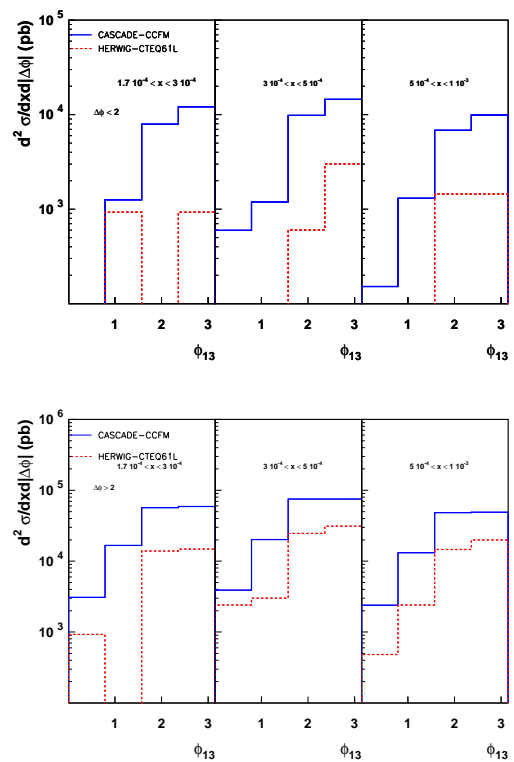

Figure 5: Cross section in the azimuthal angle between the hardest and the third jet 60, for (top) small and (bottom) large azimuthal separations between the leading jets.

CASCADE gives a good description of the measurement, and gives large differences to the collinear-based parton shower as implemented in HERWIG, particularly in the region where the azimuthal separations $\Delta \phi$ between the leading jets are small.

The $\mathrm{x}$ dependence of the gluon distribution used in the above results for the jet cross sections is shown in Fig. 6 for various scales $\mu$ and fixed $\mathrm{k}_{\perp}$.

This analysis indicates that theoretical uncertainties on jet correlations are sizeable at NLO 78 and that the $\mathrm{k}_{\perp}$ Monte-Carlo gives an improved description of multi-jets 60. compared to standard parton showers. This analysis is for DIS. However, despite the 


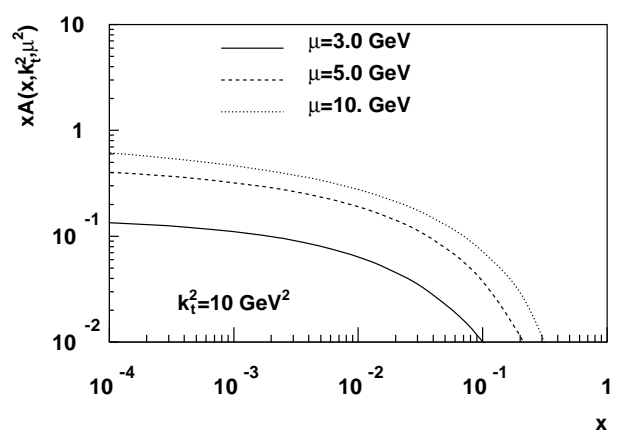

Figure 6: The $\mathrm{x}$ dependence of the unintegrated gluon distribution.

much lower energy at HERA, note that owing to the large phase space available for jet production the HERA data may be just as relevant as the Tevatron for understanding the extrapolation to the LHC of initial-state radiation effects [7].

\subsection{Forward region}

Transverse energy flow and production of jets and particles at forward rapidities are important probes of the initial-state $\mathrm{k}_{\perp} 83$. Monte-Carlo results for such observables, however, strongly depend on the details of the model used for evolution of the u-pdf's. This model-dependence is much more pronounced for forward-region observables than for the jet correlations discussed in the previous subsection. A systematic understanding of u-pdf's evolution 84 will be relevant for more precise interpretations of forward measurements. See 40 for recent work on evolution equations, including discussion of target fragmentation. We refer the reader to 17, 16, and references therein, for discussion of current forward-region results.

\section{Towards precise characteriza- tions of u-pdf's}

The possibility to turn the event generators discussed in Sec. 2 into general tools to describe hadronic final states over the whole phase space depends on theoretical progress about unintegrated parton distributions. This will involve precise characterizations of the distributions, factorization and evolution equations. In what follows we discuss recent progress in the understanding of operator matrix elements for u-pdf's and their lightcone limits.

\subsection{Gauge invariant matrix elements}

The relevance of consistent operator definitions for parton $\mathrm{k}_{\perp}$ distributions was emphasized long ago, see e.g. [5, 85]. Ensuring gauge invariance at $\mathrm{k}_{\perp} \neq 0$, however, is nontrivial. To this end the approach commonly used is to generalize the coordinatespace matrix elements that serve to identify ordinary pdf's to the case of field operators at non-lightcone distances. E.g., for quarks one has 6, 86, 87

$$
\widetilde{f}(y)=\left\langle P\left|\bar{\psi}(y) V_{y}^{\dagger}(n) \gamma^{+} V_{0}(n) \psi(0)\right| P\right\rangle .
$$

Here $\psi$ are the quark fields evaluated at distance $y=\left(0, y^{-}, y_{\perp}\right), y_{\perp}$ is in general nonzero, and $V$ are eikonal-line operators in direction $n$ required to make the matrix element gauge-invariant. The unintegrated quark distribution is obtained from the double Fourier transform in $y^{-}$and $y_{\perp}$ of $\widetilde{f}$. An extra gauge link at infinity [86] is to be taken into account in the case of physical gauge.

There are subtleties, however, to using Eq. (11) beyond tree level. As realized early on 85, parton distributions at fixed $\mathrm{k}_{\perp}$ are no longer protected by the KLN mechanism [88] against uncancelled lightcone divergences near the $\mathrm{x}=1$ endpoint 87 , 89]. It is only after supplying the above matrix element with a regularization prescription that the distribution is well-defined.

Importantly, similar to what observed in 90 for the case of the Sudakov form factor, the choice of a particular regularization method for the lightcone divergences also affects the distributions integrated over $\mathrm{k}_{\perp}$ and 
the ultraviolet subtractions. A further set of questions being studied [27, 51, 91, 92] are in fact associated with the coefficient functions that govern the expansion of unintegrated distributions in terms of ordinary ones, and the relation of the integral of u-pdf's with ordinary distributions.

The above issues can be analyzed by explicit calculation at one loop [27, 93. Expansion in powers of $y^{2}$ of the result for the coordinate-space matrix element at this order (Fig. 7) yields

$$
\begin{aligned}
& \tilde{f}_{1}(y)=\frac{\alpha_{s} C_{F}}{\pi} p^{+} \int_{0}^{1} d v \frac{v}{1-v} \\
& \times\left\{\left[e^{i p \cdot y v}-e^{i p \cdot y}\right] \Gamma\left(2-\frac{d}{2}\right)\left(\frac{4 \pi \mu^{2}}{\rho^{2}}\right)^{2-d / 2}\right. \\
& +e^{i p \cdot y v} \pi^{2-d / 2} \Gamma\left(\frac{d}{2}-2\right)\left(-y^{2} \mu^{2}\right)^{2-d / 2} \\
& +\cdots\}
\end{aligned}
$$

where $\mu$ is the dimensional-regularization scale and $\rho$ is an infrared mass regulator. The lightcone singularity $v \rightarrow 1$, corresponding to the exclusive boundary $x=1$, cancels for ordinary pdf's (first term in the right hand side of Eq. (2) ) but it is present, even at $d \neq 4$ and finite $\rho$, in subsequent terms. This implies that, using the matrix element (11), the $1 /(1-x)$ factors from real emission probabilities do not combine with virtual corrections to give $1 /(1-x)_{+}$distributions, but leave uncancelled divergences at fixed $\mathrm{k}_{\perp}$.

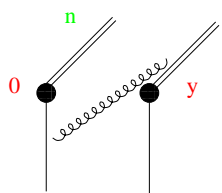

(a)

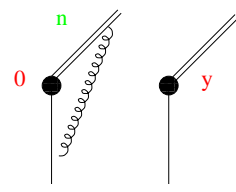

(b)
Figure 7: One-loop contributions to lightcone divergences in the matrix element (1).

Different methods have been employed in the literature to provide u-pdf's with consistent regularization for the endpoint region.
We consider these methods briefly in the next subsection.

\subsection{Cut-off vs. subtractive method}

A possible approach to treat the endpoint is to implement a cut-off by taking the eikonal line $n$ to be non-lightlike, as in the early works 85 and 94. The cut-off in $\mathrm{x}$ at fixed $\mathrm{k}_{\perp}$ is of order $1-x \geq k_{\perp} / \sqrt{4 \eta}$, where $\eta=$ $(p \cdot n)^{2} / n^{2}$ and $p$ is the incoming momentum. Evolution equations in the cut-off parameter $\eta$ are investigated in $36,85,95,96]$. A thorough analysis of factorization at leading order has recently been given in [26] based on this approach.

A potential drawback of this approach is that cut-off regularization is not very wellsuited for applications beyond the leading order. Also, as the two lightcone limits $y^{2} \rightarrow 0$ and $n^{2} \rightarrow 0$ do not commute, the integral over $\mathrm{k}_{\perp}$ of the distribution has a finite dependence on the regularization parameter $\eta$,

$$
\begin{aligned}
\int d k_{\perp} f\left(x, k_{\perp}, \mu, \eta\right) & =F(x, \mu, \eta) \\
& \neq \text { ordinary pdf }
\end{aligned}
$$

which makes the relation with the standard operator product expansion not so transparent.

An alternative route is based on the subtractive method [90, 97. In this case the direction $n$ is kept to be lightlike but the divergences are canceled by multiplicative, gauge-invariant counterterms given by vacuum expectation values of eikonal operators. The counterterms contain in general both lightlike and non-lightlike eikonals. For this reason studies of u-pdf's in this framework introduce auxiliary eikonals in direction $u=\left(u^{+}, u^{-}, 0_{\perp}\right)$ with $u^{+}$and $u^{-}$ nonzero [27, 87]. The counterterms have compact all-order expressions in coordinate space. At one loop they provide the regularization of the $\mathrm{x} \rightarrow 1$ endpoint through a $\mathrm{k}_{\perp} \neq 0$ extension of the plus-distribution 
regularization, whose specific form is determined in [27].

The subtractive method is more systematic than the cut-off, and likely more suitable for using unintegrated parton distributions at subleading-log level. In particular, the specific choice of the counterterms 27 is such that the dependence on the non-lightlike eikonal $u$ cancels in the matrix element at $y_{\perp}=0$, corresponding to the ordinary pdf. The subtractive method may be helpful for global NLO analyses incorporating Sudakov resummation 98, 99, and construction of parton-shower algorithms beyond leading order 191, 100. See 101, 102, 103 for studies of subtractive methods similar to those of 90,97 in relation to effective-theory techniques.

\subsection{Further issues in higher order}

Further issues arise in higher order. Nonuniversality of $\mathrm{k}_{\perp}$-dependent parton distributions [28] has recently been studied [29-32] in the hadroproduction of back-to-back high$p_{t}$ particles. Potential factorization-breaking effects from soft gluons coupling initial and final states are shown in Fig. 8 30. These would enter at a high order of perturbation theory $\left(\mathrm{N}^{3} \mathrm{LO}\right.$ correction to dihadron production). Although the role of these corrections is yet to be fully established, it is interesting that Coulomb/radiative mixing terms also appear to break color coherence [104 in high-order contributions to cross sections for dijets with a gap in rapidity. A satisfactory understanding of factorization should likely require a full clarification of these issues.

\section{Conclusion}

Monte-Carlo event generators are being developed based on gauge-invariant definition of u-pdf's at small x (Sec. 2.1). Compared to standard parton shower approaches, these Monte-Carlos have the advantage of taking into account QCD initial-state radiation pro-

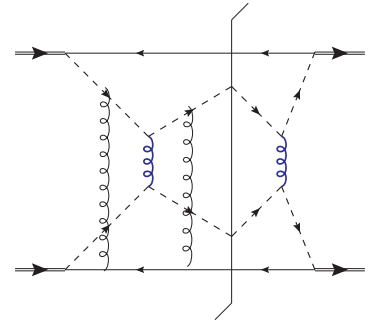

Figure 8: Soft gluon exchange with spectator partons.

cesses that depend on the large- $\mathrm{k}_{\perp}$ tail of partonic distributions and matrix elements.

Such processes are likely to be relevant to the simulation of complex final states characterized by multiple hard scales. An example is the DIS multi-jet correlations discussed in Sec. 2.3. But we expect analogous considerations to apply for multi-jet final states in hadron-hadron collisions at LHC energies.

$\mathrm{k}_{\perp}$ Monte-Carlos are currently being tuned and validated using HERA and Tevatron data, as discussed in Secs. 2.2 and 2.3. Determinations of the unintegrated gluon density are performed.

It will be important to extend the notion of u-pdf's beyond $\mathrm{x} \ll 1$ (Sec. 3 ) to turn the above event generators into general-purpose tools.

This involves a challenging theoretical program, raising new questions on operator matrix elements (Sec. 3.1), factorization and lightcone divergences (Sec. 3.2), and possibly new Coulombic effects (Sec. 3.3).

\section{References}

[1] Yu.L. Dokshitzer, D. Diakonov and S.I. Troyan, Phys. Rept. 58 (1980) 269.

[2] J.C. Collins, D.E. Soper and G. Sterman, Nucl. Phys. B250 (1985) 199.

[3] L.V. Gribov, E.M. Levin and M.G. Ryskin, Phys. Rept. 100 (1983) 1.

[4] S. Catani, M. Ciafaloni and F. Hautmann, Phys. Lett. B242 (1990) 97.

[5] S.J. Brodsky and G.P. Lepage, in Perturbative Quantum Chromodynamics, ed. A.H. Mueller, World Scientific 1989, p. 93. 
[6] P. Mulders and R.D. Tangerman, Nucl. Phys. B461 (1996) 197; D. Boer and P. Mulders, Phys. Rev. D 57 (1998) 5780.

[7] S. Alekhin et al., hep-ph/0601012 Proceedings of the Workshop "HERA and the LHC".

[8] S. Catani, M. Ciafaloni and F. Hautmann, Nucl. Phys. B366 (1991) 135.

[9] G. Marchesini and B.R. Webber, Nucl. Phys. B386 (1992) 215.

[10] R.K. Ellis, F. Hautmann and B.R. Webber, Phys. Lett. B 348 (1995) 582.

[11] M. Ciafaloni, D. Colferai, G.P. Salam and A.M. Stasto, JHEP 0708 (2007) 046.

[12] M. Ciafaloni, D. Colferai, G.P. Salam and A.M. Stasto, Phys. Lett. B 635 (2006) 320.

[13] G. Altarelli, R.D. Ball and S. Forte, Nucl. Phys. B742 (2006) 1.

[14] C.D. White and R.S. Thorne, Phys. Rev. D 75 (2007) 034005.

[15] R.D. Ball, arXiv:0708.1277

[16] J.R. Andersen et al., Eur. Phys. J. C48 (2006) 53.

[17] S. Höche, F. Krauss and T. Teubner, arXiv:0705.4577

[18] K. Golec-Biernat, S. Jadach, W. Placzek, P. Stephens and M. Skrzypek, hep-ph/0703317

[19] H. Jung, Mod. Phys. Lett. A19 (2004) 1.

[20] H. Jung, Comput. Phys. Commun. 143 (2002) 100.

[21] H. Jung and G.P. Salam, Eur. Phys. J. C19 (2001) 351.

[22] L. Lönnblad and M. Sjödahl, JHEP 0402 (2004) 042; JHEP 0505 (2005) 038.

[23] G. Gustafson, L. Lönnblad and G. Miu, JHEP 0209 (2002) 005.

[24] Y. Hatta, E. Iancu and A.H. Mueller, arXiv:0710.2148

[25] F. Gelis, T. Lappi and R. Venugopalan, arXiv:0708.0047

[26] J.C. Collins, T.C. Rogers and A.M. Stasto, arXiv:0708.2833

[27] F. Hautmann, Phys. Lett. B 655 (2007) 26.

[28] C.J. Bomhof, P.J. Mulders and F. Pijlman, Eur. Phys. J. C47 (2006) 147; Phys. Lett. B 596 (2004) 277.

[29] C.J. Bomhof and P.J. Mulders, arXiv:0709.1390

[30] J.C. Collins, arXiv:0708.4410.

[31] Y. Koike, W. Vogelsang and F. Yuan, arXiv:0711.0636
[32] W. Vogelsang and F. Yuan, Phys. Rev. D 76 (2007) 094013.

[33] J.W. Qiu, W. Vogelsang and F. Yuan, Phys. Rev. D 76074029.

[34] X. Ji, J.W. Qiu, W. Vogelsang and F. Yuan, Phys. Rev. Lett. 97 (2006) 082002.

[35] K. Goeke, S. Meissner and A. Metz, Phys. Rev. D 76 (2007) 034002.

[36] P. Chen, A. Idilbi and X. Ji, Nucl. Phys. B763 (2007) 183.

[37] I.O. Cherednikov and N.G. Stefanis, arXiv:0710. 1955; arXiv:0711.1278

[38] D.S. Hwang and D.S. Kim, arXiv:0710.2672

[39] J. Chay, arXiv:0711.4295.

[40] F. Ceccopieri and L. Trentadue, arXiv:0706. 4242 .

[41] L.N. Lipatov, Sov. J. Nucl. Phys. 23 (1976) 338; E.A. Kuraev, L.N. Lipatov and V.S. Fadin, Sov. Phys. JETP 45 (1977) 199.

[42] I.I. Balitsky and L.N. Lipatov, Sov. J. Nucl. Phys. 28 (1978) 822.

[43] M. Ciafaloni, Nucl. Phys. B296 (1988) 49.

[44] S. Catani, F. Fiorani and G. Marchesini, Nucl. Phys. B336 (1990) 18.

[45] B. Andersson, G. Gustafson and J. Samuelsson, Nucl. Phys. B467 (1996) 443.

[46] T. Sjöstrand, S. Mrenna and P. Skands, JHEP 0605 (2006) 026.

[47] G. Corcella et al., JHEP 0101 (2001) 010.

[48] S. Höche, F. Krauss, N. Lavesson, L. Lönnblad, M. Mangano, A. Schälicke and S. Schumann, hep-ph/0602031

[49] G. Gustafson, talk at the Workshop "HERA and the LHC", Hamburg, March 2007.

[50] See talks by S. Gieseke and T. Sjöstrand at Workshop on Multiparton Interactions, Hamburg, May 2007.

[51] A.D. Martin, M.G. Ryskin and G. Watt, Eur. Phys. J. C31 (2003) 73.

[52] M.A. Kimber, A.D. Martin and M.G. Ryskin, Phys. Rev. D 63 (2001) 114027.

[53] S. Catani and F. Hautmann, Nucl. Phys. B427 (1994) 475; Phys. Lett. B 315 (1993) 157.

[54] M. Ciafaloni and D. Colferai, JHEP 0509 (2005) 069.

[55] A. Gawron, J. Kwiecinski and W. Broniowski, Phys. Rev. D 68 (2003) 054001.

[56] B.A. Kniehl, V.A. Saleev and D.V. Vasin, Phys. Rev. D 74 (2006) 014024; Phys. Rev. D 73 (2006) 074022. 
[57] A. Szczurek, A. Rybarska and G. Slipek, Phys. Rev. D 76 (2007) 034001.

[58] H. Jung, A.V. Kotikov, A.V. Lipatov and N.P. Zotov, arXiv:0706.3793

[59] H. Jung, hep-ph/0411287

[60] F. Hautmann and H. Jung, preprint OUTP-07$12-\mathrm{P}$, in preparation.

[61] H1 Coll. (A. Aktas et al.), Eur. Phys. J. C33 (2004) 477

[62] M. Hansson and H. Jung, arXiv:0707.4276

[63] CDF Coll. (A. Abulencia et al.), Phys. Rev. D 75 (2007) 092006.

[64] F. Hautmann, Phys. Lett. B 535 (2002) 159.

[65] A. Kulesza, G. Sterman and W. Vogelsang, Phys. Rev. D 69 (2004) 014012.

[66] A. Kulesza, G. Sterman and W. Vogelsang, Acta Phys. Polon. B 34 (2003) 5503.

[67] C. Balazs, M. Grazzini, J. Huston, A. Kulesza and I. Puljak, hep-ph/0403052

[68] S. Alekhin et al., hep-ph/0601013

[69] A. Gawron and J. Kwiecinski, Phys. Rev. D 70 (2004) 014003.

[70] A.V. Lipatov and N.P. Zotov, Eur. Phys. J. C44 (2005) 559.

[71] M. Luszczak and A. Szczurek, Eur. Phys. J. C46 (2006) 123.

[72] S. Heinemeyer, V.A. Khoze, M.G. Ryskin, W.J. Stirling, M. Tasevsky and G. Weiglein, arXiv:0708.3052

[73] A. De Roeck, V.A. Khoze, A.D. Martin, R. Orava and M.G. Ryskin, Eur. Phys. J. C25 (2002) 391.

[74] R.S. Pasechnik, A. Szczurek and O.V. Teryaev, arXiv:0709.0857

[75] J. Bartels, A. Sabio Vera and F. Schwennsen, arXiv:0709.3249 JHEP 0611 (2006) 051.

[76] F. Schwennsen, hep-ph/0703198

[77] J.R. Andersen, V. Del Duca, S. Frixione, F. Maltoni, C.R. Schmidt and W.J. Stirling, hep-ph/0109019

[78] Zeus Coll. (S. Chekanov et al.), Nucl. Phys. B 786 (2007) 152

[79] Y. Delenda, R. Appleby, M. Dasgupta and A. Banfi, JHEP 0612 (2006) 044.

[80] A. Banfi and M. Dasgupta, JHEP 0401 (2004) 027.

[81] Z. Nagy and Z. Trocsanyi, Phys. Rev. Lett. 87 (2001) 082001.

[82] Y. Delenda, arXiv:0706.2172
[83] See e.g. B.R. Webber, hep-ph/9510283 in Proceedings of DIS95 (Paris, 1995).

[84] See for instance the discussion in J.C. Collins, hep-ph/0106126 in Proceedings of DIS01 (Bologna, 2001).

[85] J.C. Collins, in Perturbative Quantum Chromodynamics, ed. A.H. Mueller, World Scientific 1989, p. 573.

[86] A.V. Belitsky, X. Ji and F. Yuan, Nucl. Phys. B656 (2003) 165.

[87] J.C. Collins, Acta Phys. Polon. B 34 (2003) 3103.

[88] T. Kinoshita, J. Math. Phys. 3 (1962) 650; T.D. Lee and M. Nauenberg, Phys. Rev. 133 (1964) 1549.

[89] S.J. Brodsky, D.S. Hwang, B.Q. Ma and I. Schmidt, Nucl. Phys. B593 (2001) 311.

[90] J.C. Collins and F. Hautmann, Phys. Lett. B 472 (2000) 129.

[91] J.C. Collins and X. Zu, JHEP 0503 (2005) 059.

[92] S. Catani, M. Ciafaloni and F. Hautmann, Phys. Lett. B307 (1993) 147.

[93] F. Hautmann, arXiv:0708.1319

[94] G.P. Korchemsky, Phys. Lett. B 220 (1989) 62.

[95] G.P. Korchemsky and G. Marchesini, Phys. Lett. B 313 (1993) 433.

[96] X. Ji, J. Ma and F. Yuan, Phys. Rev. D 71 (2005) 034005, JHEP 0507 (2005) 020.

[97] J.C. Collins and F. Hautmann, JHEP 0103 (2001) 016.

[98] C.P. Yuan, talk at DIS07 Workshop, Munich, April 2007: http://indico.cern.ch/contributionDisplay. py? contribId=251\&session Id=8\&conf $I d=9499$

[99] P.M. Nadolsky, N. Kidonakis, F.I. Olness and C.P. Yuan, Phys. Rev. D 67 (2003) 074015; R. Brock, F. Landry, P.M. Nadolsky and C.P. Yuan, Phys. Rev. D 67 (2003) 073016.

[100] C.W. Bauer and M.D. Schwartz, Phys. Rev. D 76 (2007) 074004.

[101] A.V. Manohar and I.W. Stewart, Phys. Rev. D 76 (2007) 074002.

[102] C. Lee and G. Sterman, Phys. Rev. D 75 (2007) 014022.

[103] A. Idilbi and T. Mehen, arXiv:0707.1101 Phys. Rev. D 75 (2007) 114017.

[104] J.R. Forshaw, A. Kyrieleis and M.H. Seymour, JHEP 0608 (2006) 059. 